Published in final edited form as:

Shock. 2016 November ; 46(5): 465-467. doi:10.1097/SHK.0000000000000735.

\title{
WHAT'S NEW IN SHOCK, NOVEMBER 2016?
}

\author{
Philip A. Efron, MD \\ Departments of Surgery, Anesthesiology, Aging and Geriatric Research, and Molecular Genetics \\ and Microbiology
}

The journal Shock is unique in that it reports on all aspects of inflammation, injury and sepsis - in a sense, "Shock is where the action is!" This month's journal only serves to emphasize how Shock remains at the forefront of international clinical and scientific research. Be it a review article or basic science manuscript, the November 2016 issue of Shock is a must read for all individuals interested in the field.

This month's journal begins with a very thorough and complete review of plasma transfusion from our colleagues on the west coast of the United States (1). Understanding the benefits versus the detriments of transfusion has become a key aspect of daily bedside medicine. In fact, transfusion is now tracked as a 'best practice measure' by many national organizations. Watson, et al's essential review of the medical use of plasma, from its origins to its ongoing research (1) is both opportune and beneficial. In addition to summarizing all of the key literature and studies regarding the use of plasma for shock, it discusses the various formulations of plasma that are currently being used or in development (1). Certainly this article will be in the library of every critical care resident for the next decade.

As Shock is the official journal of a consortium of international societies, it is of no surprise that the next article illustrates some exceptional work from South America. Dr. Filho, et al have produced some very timely work regarding blood lactate levels and sepsis (2). The Third International Consensus Definitions for Sepsis and Septic Shock (Sepsis-3) has defined septic shock as the requirement for a vasopressor (in the absence of hypovolemia) to maintain a minimum mean arterial pressure of $65 \mathrm{mmHg}$ and a serum lactate level greater than $2 \mathrm{mmol} / \mathrm{L}(>18 \mathrm{mg} / \mathrm{dL}$ ) (3). With this in mind, abnormal lactate levels above a level of $2 \mathrm{mmol} / \mathrm{L}$ are now more relevant than ever, as presented by Filho and colleagues, who were able to use an Emergency Room admission lactate cutoff of $2.5 \mathrm{mmol} / \mathrm{L}$ to predict 28 day mortality, and this had a receiver operator curve of 0.70 (2). Although Filho et al.'s work is a retrospective analysis, this real world study presents a simple, clinically relevant test that can be utilized for predicting mortality in septic patients with organ dysfunction or pressor requirements (2).

Not unexpectedly, Dr. Herndon's group continues to lead the way in demonstrating the effectiveness of interventional therapies that are United States Food and Drug Administration approved as well as inexpensive. It goes against the better judgement of any clinician to give a medication that potentially lowers blood pressure in the patient in the acute phase of an inflammatory response. However, Dr. Wurzer, et al have demonstrated that giving propanolol in burned children is not only safe but potentially helpful (4). The medication reduced cardiogenic stress without adversely affecting other multiple important 
parameters (4). This includes, but is not limited to: peripheral oxygen delivery, events of lactic acidosis, wound healing, and mortality (4). Clearly, Dr. Wurzer and colleagues are part of a select few investigators with the remarkable ability to see beyond many clinicians' trepidation to utilize agents such as propanolol in the initial phase of inflammation and shock to help improve outcomes $(5,6)$.

Keeping with the theme of top notch work accomplished in both the burn population as well as around the world, Dr. Lopez-Rodriguez, et al have continued their analysis of their singlecenter, prospective, randomized, double-blind clinical trial on the effect of selective decontamination of the digestive tract (SDD) in severe burn patients (7). 'No organ is an island,' and this is certainly true of the intestines in the acute and sub-acute phases of shock, trauma and burn. After previously demonstrating that SDD can improve mortality after burns, the authors have illustrated that this simple intervention was independently associated with a reduction in organ dysfunction in severely burned patients, which also suggests a modulatory role of SDD on the inflammatory response (7). And what was able to induce this huge impact? The answer is the early and temporary use of non-proprietary antibiotics, such as polymyxin, amphotericin B and cefotaxime (7). Again, an inexpensive and safe intervention is demonstrated to have an impact, this time influencing the host as well as the microbiome of these very sick patients (8).

Not to be superseded, French investigators have also analyzed an inexpensive drug that has been debated for decades in sepsis. I am, of course, referring to steroids! Dr. Herve, et al conducted a multicenter, prospective, randomized, double-blind, pilot study comparing two low dose regimens of hydrocortisone: $200 \mathrm{mg}$ versus $300 \mathrm{mg}$ (9). Their results have illustrated, like many of the recent sepsis prospective trials in sepsis, that it's not so much the specifics of the intervention, such as the total dose, but the early recognition of the need for a therapy along with its timely institution that is most important to the patient's outcome (9). Thus, the authors found no difference in 28 day mortality between the groups - however, the analysis may lead to important further weight based steroid experimentation, as well reviews of sepsis persistence and etomidate use in these patients (9).

Leelahavanichkul, et al have made further progress in the field of practical predictive medicine (10). This work from Thailand looked at serum $(1 \rightarrow 3)$-b-D-glucan (BG) in not just septic mice, but septic humans (10). BG is a key structural polysaccharide of the cell wall of most fungi and the authors have revealed that it can be used as a marker of gastrointestinal leakage and sepsis, even during/after bacterial infections (10). As clinicians are moving towards precision medicine (11), this type of biomarker will prove vital as scientists tailor therapy only to those individuals that require intervention.

It's impossible to investigate inflammation without considering toll-like receptors (TLR), and when you combine Dr. Billiar's laboratory and the journal Shock, outstanding work is always revealed. That tradition continues this month as Korff, Scott, Billiar, et al use clever investigative basic science, including knock out and chimera mice and a clinically relevant model of hemorrhagic shock (12), to further our understanding of the role of TLR in inflammation. Their work illustrated that TLR2 regulates both bone marrow and non-bone marrow derived cells' ability to affect inflammation, and more importantly, organ failure 
(12). Interestingly, the mechanism of this was in conjunction with TLR4 (12). It is only through clinically relevant work like this that progress will be made to enable future physicians to immunomodulate the human patient after trauma and hemorrhagic shock.

As a global journal, revealing and analyzing cutting edge technology has always been a vital component of the journal Shock. Thus, in this November's issue the editors are very proud to publish the work of Dr. Lane Smith and the collaborative team at Wake Forrest.

Photoacoustic (PA) imaging, is a technology that evaluates both tissue structure and function through ultrasound and laser energy (13). The investigators have been able to illustrate that PA is able to measure, in real-time, oxygen saturation in the macro and microcirculation during acute hypoxia (13). Anyone involved in research regarding shock will need to be familiar with this technology, as its future use, in combination with other agents, will likely become common place in the critically ill (13).

Clinical relevance does not mean abandoning basic science at its most fundamental level. Kidney injury is a key driver of poor patient outcomes - much more than previously realized. Dr. Wang and colleagues from China have performed some very elegant basic research regarding myofibrillogenesis regulator 1 (MR-1) in a clinically relevant murine model of renal ischemia/reperfusion injury (I/R) (14). MR-1 is a mitochondrial-targeted protein, and the researchers demonstrated that through the recruitment of phosphatidylinositol 3 kinasedependent phosporylated-Akt to the mitochondria, MR-1 was able to protect the kidney from I/R injury by inhibiting mitochondrial permeability transition pore opening and maintaining mitochondrial integrity (14). The various methodologies conducted by the investigators in this study are impressive, and this work has the capacity to introduce new therapeutics to the realm of shock research (14).

Returning to the investigation of burn injury, Dr. Caldwell's laboratory has revealed another important aspect to the treatment of depression (15). Although depression is not considered normally in the area of shock research, depression and medication for its treatment are fairly prevalent in the United States. Dr. Johnson, et al were able to determine that amitriptyline, a tricyclic antidepressant that inhibits acid sphingomyelinase, had significant effects on the immunity of host after burn injury (15). This included a reduction in lymphocyte precursors, lymphocyte numbers and neutrophil recruitment (15). Clearly, their work suggests that future precision medicine for inflammation, injury and infection will require consideration of the patient's 'medication profile.'

Which leads to the concept that precision medicine will also need to take into consideration a patient's diet with the treatment of, let's say, of cardiovascular disease. Although debated to some extent, patients whose lifestyles incorporate certain seeds or fish are much less likely to succumb to certain pathology, such as acute myocardial infarctions. However, the medical practitioner is not able to pre-emptively create such environment when their patient arrives at the emergency room. Interestingly, Burban et al have determined that one single intravenous omega-3 bolus before reperfusion in a clinically relevant rat model of I/Rinduced shock was able to improve multiple parameters (16). This included an increased mean arterial pressure and carotid blood flow as well as decreased cardiac troponin levels (16). This improvement in blood pressure and vasoreactivity could have significant 
repercussions regarding the treatment of acute coronary syndromes, and again the work in this issue reveals possible therapies that are both inexpressive and safe (16)!

The need to improve cardiac function and outcomes is not limited to myocardial infractions, though. Dr. Li, et al have investigated the well-known cardio-dysfunction induced by severe sepsis and septic shock. Translating work done in cardiac I/R, the authors were able to conduct research on intermedin, a calcitonin related peptide, using the cecal ligation and puncture murine sepsis model (17). Early and late administration of the compound, specifically intermedin 1-53, to septic mice improved heart function, tissue oxygenation/ perfusion and survival (17). This was in part through the Rho kinase phosphorylation pathway and by increasing intracellular calcium (17).

As a journal of world-wide importance, it is of no surprise that notable work is being published in this issue Shock regarding melioidosis. This is an infection with Burkholderia pseudomallei that is relatively common in Southeast Asia and Northern Australia with significant morbidity and mortality. Dr. Weehuiz has produced some impressive data with a collaborative international laboratory group regarding the use of a monoclonal anti-IL-1b antibody in this condition (18). Although treatment with such compounds in the past failed to improved outcomes, application of anti-IL1b antibodies could be effective in more select patient population, such as those suffering from melioidosis (18). This research was able to demonstrate that their intervention were able to affect the inflammasome and improve the host's response to the bacterium B. pseudomallei(18).

Taiwanese investigators have made some 'in-roads' regarding our understanding of the acute respiratory distress syndrome, as displayed by the work of Day, et al. Using a rat model of the Acute Respiratory Distress Syndrome (ARDS) combined with intraperitoneal lipopolysaccharide injection, the authors were able test the hypothesis that preactivated and disaggregated shape-changed platelets could attenuate lung injury (19). Their intervention was able to improve the outcomes of the host on multiple levels, including, but not limited to, histological, cellular and molecular results (19). This work has improved not only our understanding of the relationship of pathology of ARDS, but the anti-inflammatory and antioxidative properties of preactivated and disaggregated shape-changed platelets, which may be considered as a future therapeutic in a disease process that has few current interventions besides supportive care (19).

Finally, in an article that combines some of the themes investigated by other scientists in this issue (sepsis, ARDS and a single injection of nutritional compound), Dr. Yeh and colleagues have eloquently demonstrated that glutamine can modify progenitor cell and lung injury in mice exposed to severe infection (20). Their basic science work revealed that an intravenous injection of glutamine after cecal ligation and puncture could promote the mobilization of endothelial progenitor cells, in part due to the release of C-X-C motif chemokine 1, vascular endothelial growth factor and nitric oxide (20). These effects are associated with improved vascular function, ameliorated inflammation and reduced damage of lung tissues (20). Although the use of glutamine in the critically ill is currently debated, the Shock journal has never shied away from contentious issues that require further investigation in order to elucidate appropriate answers for the scientific and medical community! 
Based on this issue and the ten issues before it in 2016, there can be no doubt that the journal Shock has led the way this year in reporting the best research, both laboratory and clinical, regarding inflammation, injury and sepsis. For several decades now, this journal has truly served its readers around the world.

\section{References}

1. Watson JJJ, Pati S, Schreiber MA. Plasma transfusion: History, current realities, and novel improvements. Shock. 2016; 46

2. Filho RR, Rocha LL, Corrêa TD, Pessoa CMS, Colombo G, Assuncão MSC. Blood lactate levels cutoff and mortality prediction in sepsis-time for a reappraisal? A retrospective cohort study. Shock. 2016; 46

3. Singer M, Deutschman CS, Seymour CW, Shankar-Hari M, Annane D, Bauer M, Bellomo R, Bernard GR, Chiche JD, Coopersmith CM, Hotchkiss RS, Levy MM, Marshall JC, Martin GS, Opal SM, Rubenfeld GD, van der Poll T, Vincent JL, Angus DC. The Third International Consensus Definitions for Sepsis and Septic Shock (Sepsis-3). JAMA. 2016; 315:80-10.

4. Wurzer P, Branski LK, Clayton RP, Hundeshagen G, Forbes AA, Voigt CD, Andersen CR, Kamolz L-P, Woodson LC, Suman OE, Finnerty CC, Herndon DN. Propranolol reduces cardiac index but does not adversely affect peripheral perfusion in severely burned children. Shock. 2016; 46

5. Morelli A, Ertmer C, Westphal M, Rehberg S, Kampmeier T, Ligges S, Orecchioni A, D’Egidio A, D'Ippoliti F, Raffone C, Venditti M, Guarracino F, Girardis M, Tritapepe L, Pietropaoli P, Mebazaa A, Singer M. Effect of heart rate control with esmolol on hemodynamic and clinical outcomes in patients with septic shock: a randomized clinical trial. JAMA. 2013; 310:1683-91. [PubMed: 24108526]

6. Novotny NM1, Lahm T, Markel TA, Crisostomo PR, Wang M, Wang Y, Ray R, Tan J, Al-Azzawi D, Meldrum DR. beta-Blockers in sepsis: reexamining the evidence. Shock. 2009; 31:113-9. [PubMed: 18636043]

7. López-Rodríguez L, de la Cal MA, García-Hierro P, Herrero R, Martins J, van Saene HKF, Lorente JA. Selective digestive decontamination attenuates organ dysfunction in critically ill burn patients. Shock. 2016; 46

8. Krezalek MA1, DeFazio J, Zaborina O, Zaborin A, Alverdy JC. The Shift of an Intestinal "Microbiome" to a "Pathobiome" Governs the Course and Outcome of Sepsis Following Surgical Injury. Shock. 2016; 45:475-82. [PubMed: 26863118]

9. Hervé H, Rémy B, Gentilhomme A, Francois C-GJ, Freche A, Kaidomar M, Bernard G, Pradier C, Dellamonica J, Bernardin G. Effects of increasing hydrocortisone to $300 \mathrm{mg}$ per day in the treatment of septic shock: A pilot study. Shock. 2016; 46

10. Leelahavanichkul A, Worasilchai N, Wannalerdsakun S, Jutivorakool K, Somparn P, IssaraAmphorn J, Tachaboon S, Srisawat N, Finkelman M, Chindamporn A. Gastrointestinal leakage detected by serum $(1 \rightarrow 3)$ - $\beta$-D-glucan in mouse models and a pilot study in patients with sepsis. Shock. 2016; 46

11. Mathias B, Lipori G, Moldawer LL, Efron PA. Integrating "big data" into surgical practice. Surgery. 2016; 159:371-4. [PubMed: 26603852]

12. Korff S, Loughran P, Cai C, Fan J, Elson G, Shang L, Pires SS, Lee YS, Guardado J, Scott M, Billiar TR. TLR2 on bone marrow and non-bone marrow derived cells regulates inflammation and organ injury in cooperation with TLR4 during resuscitated hemorrhagic shock. Shock. 2016; 46

13. Smitih L, Varagic J, Yamaleyeva L. Photoacoustic imaging for the detection of hypoxia in the rat femoral artery and skeletal muscle microcirculation. Shock. 2016; 46

14. Wang X-R, Ding R, Tao T-Q, Mao H-M, Liu M, Xie Y-S, Liu X-H. Myofibrillogenesis regulator 1 rescues renal ischemia/reperfusion injury by recruitment of P13K -dependent P-AKT to mitochondria. Shock. 2016; 46 
15. Johnson BL III, Rice TC, Xia BT, Boone KI, Green EA, Gulbins E, Caldwell CC. Amitriptyline usage exacerbates the immune suppression following burn injury. Shock. 2016; 46

16. Burban M, Meyer G, Olland A, Séverac F, Yver B, Toti F, Schini-Kerth V, Meziani F, BoisraméHelms J. An intravenous bolus of EPA:DHA 6:1 protects against myocardial ischemia-reperfusioninduced shock. Shock. 2016; 46

17. Li T, Yu Z, Wu H, Wu Y, Zhang J, Peng X, Zang J, Xiang X, Liu L. Beneficial effect of intermedin 1-53 in septic shock rats: Contributions of Rho kinase and BKCA pathway-mediated improvement in cardiac function. Shock. 2016; 46

18. Weehuizen TAF, Lankelma JM, De Jong HK, De Boer OJ, Roelofs JJTH, Day NPJ, Gram H, De Vos AF, Wiersinga WJ. Therapeutic administration of a monoclonal anti-IL- $1 \beta$ antibody protects against experimental melioidosis. Shock. 2016; 46

19. Day Y-K, Chen K-H, Chen Y-L, Huang T-H, Sung P-H, Lee F-Y, Chen C-H, Chai H-T, Yin T-C, Chiang H-J, Chung S-Y, Chang H-W, Yip H-K. Preactivated and disaggregated shape-changed platelets protected against acute respiratory distress syndrome complicated by sepsis through inflammation suppression. Shock. 2016; 46

20. Pai M-H, Shih Y-M, Shih J-M, Yeh C-L. Glutamine administration modulates endothelial progenitor cell and lung injury in septic mice. Shock. 2016; 46 